\title{
Harmonização e classificação toxicológica de agrotóxicos em 1992 no Brasil ea necessidade de prever os impactos da futura implantação do GHS
}

\author{
Harmonization and toxicological classification of pesticides \\ in 1992 in Brazil and the need to foresee the impacts \\ from the forthcoming introduction of GHS
}

Eduardo Garcia Garcia ${ }^{1}$

M arco Antonio Bussacos ${ }^{1}$

Frida M arina Fischer ${ }^{2}$
${ }^{1}$ Coordenação deSaúdeno Trabalho, Fundação Jorge Duprat Figueiredo de Segurança e M edicina do Trabalho - Fundacentro. Rua CapoteValente 710, Jardim Paulista. 05409-002 São Paulo SP.

edgarcia@fundacentro.gov.br ${ }^{2}$ Departamento deSaúde Ambiental, Faculdadede SaúdePública,

Universidade deSão Paulo.
Abstract In 1992, Brazil modified its toxicological classification criteria for pesticides. This modification aimed to follow the guidelines of the World Health Organization (WHO) hazard classification of pesticides. In 2002, the U nited $\mathrm{Na}$ tions adopted the "Globally H armonized System for the Classification and Labelling of Chemicals" (GHS). As a result, the WHO is reviewing its classification and Brazil will probably follow the example. Therefore it seems timely to estimate the impact of the changes in the criteria adopted in 1992 in the toxicological reclassification and labelling of the commercially available products registered at that time. It is estimated that $58.6 \%$ of the total of registered pesticides ( $74.9 \%$ liquid and $31.0 \%$ solid formulations) might have been reclassified to less hazardous classes. The hazard warnings on the labels may have caused negative consequences due to misinterpretations by farmers or rural workers. For countries already having a hazard classification system, it is therefore recommendable to estimatethe possible impact of the changes before implanting the GHS.

Key words GHS, Pesticides, Risk communication, Toxicological classification, Hazard classification, Chemicals
Resumo Em 1992, o Brasil modificou seus crité rios de classificação toxicológica de agrotóxicos adequando-os à recomendação de classificação de periculosidade da Organização M undial da Saúde (O M S) . Em 2002, o Sistema Globalmente H armonizado de Classificação e Rotulagem de Produtos Químicos(GHS) foi adotado pela Organização das Nações U nidas. Em decorrência, a OM S está adequando ao GHS sua recomendação de classificação de agrotóxicos, o que também deverá ser feito pelo Brasil. Considerou-se oportuno estimar o impacto da alteração de critérios, ocorrida em 1992, na reclassificação toxicológica dos produtos comerciais que se encontravam registrados na ocasião. Encontrou-se que $58,6 \%$ do total dos agrotóxicos então registrados ( $74,9 \%$ das formulações líquidas e 31,0\% das sólidas) podem ter sofrido reclassificação para classes toxicológicas consideradas de me nor periculosidade, sofrendo mudanças na comunicação de riscos expressa na rotulagem. I sto pode ter ocasionado conseqüências negativas devido a confusões de interpretação pel os agricultores. N os países que já dispõem de sistemas de classificação de periculosidade de agrotóxicos, como o Brasil, recomenda-se estimar, antes da implantação, os impactos das mudanças que poderão decorrer da adoção do GHS.

Palavras-chave GHS, Agrotóxicos, Comunicação de riscos, Classificação toxicológica, Classificação de periculosidade, Substâncias químicas 
Introdução

Preocupações suscitadas durante a década de 1960 quanto aos efeitos secundários do uso de agrotóxicos provocaram um grande desenvolvimento de pesquisas toxicológicas e ambientais, gerando tantos dados que a interpretação dessas informações tornou-se difícil, segundo Copplestone ${ }^{1}$, implicando regulamentações bastante distintas em diferentes países.

No início da década de 1970, a Organização Mundial da Saúde- OM S², sentindo a necessidade do estabel ecimento de critérios para interpretação de dados e proposição de medidas de controle, resolveu propor uma classificação de periculosidade à saúde que pudesse servir de guia, principalmentepara os países em desenvolvimento. Isto foi concretizado em 1975 com a adoção do documento The WHO Recommended Classification of Pesticides by Hazard pela 28a Assembléia M undial de Saúde. Em 1978, atendendo as sugestões de Estados-membros e de autoridades responsáveis por registros de pesticidas sobre a necessidade de informações adicionais, um guia contendo informações específicas dos agrotóxicos, para fins de classificação, foi produzido e passou a ser apresentado como anexo do documento original, sendo atual izado a cada dois anos.

O guia da OM S2 propõe diferentes classes de periculosidade baseadas principalmente na toxicidade aguda (D ose Letal $50 \%-\mathrm{DL}_{50}$ ) oral e dermal do ingrediente ativo (produto técnico) e das suas formulações (produtos comerciais), já que sua determinação é realizada por procedimentos padronizados pelatoxicologia edefine o valor $\mathrm{DL}_{50}$ como uma estimativa estatística do número de miligramas de tóxico por quilogramas de peso vivo requerido para matar $50 \%$ de uma grande população de animais de ensaios experimentais.

Segundo Copplestone $\mathrm{I}^{1}$, o conceito de periculosidade é introduzi do pela classificação de acordo com o estado físico (sólido ou líquido) de cada produto técnico ou formulação. Os limites das classes são baseados em escala logarítmica de valores da $\mathrm{DL}_{50}$. Valores de $\mathrm{DL}_{50}$ oral para ratos são usados, mas se 0 valor da $\mathrm{DL}_{50}$ dermal para ratos colocar um dado produto em uma classe de maior periculosidade, esse valor é o utilizado como referência para a classificação.

O objetivo básico de classificar os agrotóxicos é, segundo a OM S2, procurar distinguir entre os de maior e os de menor periculosidade e, de modo geral, tem servido para harmonizar as informações nos rótulos sobreos perigos que aquele produto pode implicar.
No Brasil, a Portaria no 2203, de 1979, já definia, a partir da classificação toxicológica, dizerese símbolos que deviam constar do rótulo para fácil identificação do grau de periculosidade e descrição das precauções necessárias para o manuseio do produto, ou seja, basicamente definindo a comunicação de riscos na rotulagem. A classe toxicológica é simbolizada por faixas coloridas que devem compor o rótulo eque são de fácil identificação. Considerando uma seqüência decrescente de toxicidade aguda, as faixas apresentam as seguintes cores: vermelho vivo para os produtos de Classe I (os mais tóxicos), amarelo intenso para os de Classe II, azul intenso para os de Classe III e verde intenso para os de Classe IV (os menos tóxicos). Essa lógica de comunicação de riscos nos rótulos se mantém até os dias atuais.

Em 1989, foi promulgada a Lei no $7802^{4}$, conhecida por "Lei dos Agrotóxicos", considerada um avanço do ponto de vista da preservação da Saúde Pública e do ambiente, sobretudo por introduzir novos instrumentos para o controle dessas substâncias, apesar de nem todos terem apresentado posteriormente a eficácia esperada, como mostrou trabalho de Garcia et al. ${ }^{5}$.

Entreas modificações estabelecidas pela nova legislação e suas regulamentações, ocorreu, em 1992, por meio da Portaria SNVS no $3^{6}$, uma redefinição dos critérios de classificação toxicológica anteriormente vigentes, definidos pela PortariaDISAD $n=4 / 80^{7}$. M antiveram-sequatro classes toxicológicas definidas principalmente pela $\mathrm{DL}_{50}$ dos produtos e também por outros dados relacionados a danos na córnea, lesões na pele e concentração letal inalatória para ratos $\left(\mathrm{CL}_{50}\right)$, preval ecendo a classificação pelo dado mais agravante. Entretanto, houve mudança nos critérios que definiam as diferentes classes.

No que se refere à $D L_{50}$, tudo indica que a regulamentação da "Lei dos Agrotóxicos" reajustou as faixas que definem as classes toxicológicas, para que ficassem semel hantes à classificação da Organização Mundial da Saúde². Segundo Copplestone ${ }^{1}$, como os valores de $\mathrm{DL}_{50}$ são contínuos, háum grandenúmero de opções para a escolha das faixas que definem as diferentes classes, o que permite essa redefinição de critérios. Essas mudanças não apenas implicaram que os novos produtos registrados fossem avaliados e classificados segundo os novos critérios, como também levaram à reclassificação toxicológica produtos que já se encontravam registrados naquele momento.

Por outro lado, independentemente dessas alterações na regulamentação brasileira, também 
em 1992, a Conferência das Nações Unidas para o M eio Ambiente e o Desenvolvimento propôs, no Capítulo 19 da Agenda 21, a criação de um sistema harmonizado de classificação e rotulagem de produtos químicos que pudesse ser globalmente implantado.

Em dezembro de 2002, foi adotado pelas Nações U nidas ${ }^{8}$ o Sistema Globalmente Harmonizado de Classificação e Rotulagem de Produtos Químicos (Globally Harmonized System of Classification and Labelling of Chemicals - GHS), com a perspectiva de construir um sistema simples de classificação de substâncias químicas, de acordo com sua periculosidade, e de comunicação de informações intrínsecas aos produtos químicos: suas propriedades e precauções para o uso informadas por meio de rotulagem e de Fichas de Informação de Segurança de Produto Químico (safety data sheets - SDS).

O GHS leva em consideração o uso de substâncias químicas em geral, não se atendo especificamente aos agrotóxicos, embora englobandoos. Tanto o GHS como a classificação da OM S depericulosidade dos agrotóxicos propõem classificação e rotulagem de produtos químicos, porém apresentam diferenças entre si. I Isso levou a $\mathrm{OM} \mathrm{S}^{9}$ a informar que sua classificação de agrotóxicos se encontra em processo de revisão para se adequar aos critérios do GHS.

É certo que a OM S procurará fazer essa adequação da melhor forma possível, procurando evitar conseqüências indesejáveis que possam decorrer da mesma. No entanto, seria importante identificar problemas potenciais a serem levados em consideração no processo de implantação do GHS, em especial no caso da harmonização da classificação de agrotóxicos, sobretudo nos países que já contam com sistemas de classificação, como no caso do Brasil.

Com esse propósito, embora ocorrida em 1992, considerou-se oportuno estimar o impacto da mudança dos critérios de classificação toxicológica promovida pela regulamentação da"Lei dos Agrotóxicos", Portaria SNVS nㅇ 3/926, na reclassificação dos produtos comerciais que se encontravam registrados à época da al teração e discutir as possíveis conseqüências dessas mudanças na comunicação de riscos expressa nos rótulos, assim como possíveis implicações na saúde do trabaIhador quelida com esses produtos. Entendeu-se que a identificação desses aspectos poderia contribuir para uma melhor preparação da nova mudança que deverá ocorrer noscritérios declassificação e rotulagem dos agrotóxicos, agora para atender ao definido pelo GHS.

\section{M étodos}

Para identificar as mudanças ocorridas na classificação toxicológica eavaliar seus impactos, fezse necessário consultar várias fontes, sobretudo pela dificuldade em obter as informações necessárias ao estudo. Desatualização, discrepâncias entre fontes efalta de dados, entre outros aspectos, impossibilitaram o uso de apenas uma fonte edeterminaram a necessidade de formar um banco de dados. Utilizou-se o software EPI INFO ${ }^{10}$ para a estruturação do banco. A alimentação dos dados foi feita por apenas uma pessoa, evitando duplicidade de interpretações. M ecanismos de checagem de entrada de dados disponíveis no softwareforam empregados para controlar eventuais erros de digitação.

0 banco foi formado com 584 registros, cada um contendo:

- marca comercial;

. ingrediente(s) ativo(s) que compõe(m) o produto comercial;

. concentração do(s) ingrediente(s) ativo(s) que compõe $(\mathrm{m})$ o produto comercial;

. estado físico do produto (sólido ou líquido); - rota de exposição dos ensaios toxicológicos de $\mathrm{DL}_{50}$ (oral, dermal);

- Classe toxicológica anterior à Portaria n $3 /$ 92, informada pelo Compêndio de Defensivos Agrícolas $^{11}$, 3a edição, 1990;

- Classe toxicológica posterior à Portaria $n^{\circ} 3 /$ 92, informada pelo programa AGROFIT/98 ${ }^{12}$, do M inistério da Agricultura, complementado com informações do programa AGROTIS ${ }^{13}$ (atualização defevereiro/2000);

- Classe toxicológica posterior à Portaria n ${ }^{\circ} 3 /$ 92, informada pelo M inistério da Saúde (arquivo disponibilizado por correio eletrônico procedente desteórgão, em 18/09/2000);

. $\mathrm{DL}_{50}$ dos produtos comerciais registrados, obtida a partir de duas fontes: informada pelo programaAGROTIS ${ }^{13}$ eestimada de acordo com metodologia da $\mathrm{OM} \mathrm{S}^{2,14}$;

- Classe toxicológica anterior e posterior à Portaria $n^{\circ} 3 / 92$, determinada de acordo com metodologia da $\mathrm{OMS} \mathrm{S}^{2,14}$.

A avaliação estimativa do impacto da mudança na classificação (reclassificação) toxicológica dos produtos comerciais registrados foi obtida como segue:

a) devido às dificuldades para obtenção de informações referentes aos produtos que se encontravam registrados no momento das alterações dos critérios da classificação toxicológica, para fins deste estudo, foram considerados pro- 
dutos comerciais que sofreram alteração de classe toxicológica aqueles que constavam do Compêndio de D efensivos A grícolas ${ }^{11}$ e cuja $\mathrm{DL}_{50}$ do ingrediente ativo constava do documento The WHO recommended classification of pesticides by hazard and guidelines to classification 1990-19912.

b) devido à desatual ização e diferenças entre as fontes de informação que deveriam conter a classe toxicológica dos produtos comerciais e considerando que, segundo Copplestone ${ }^{1}$, se não houver dados detestes específicos, a $\mathrm{DL}_{50}$ da formulação (produto comercial) pode ser estimada a partir dos dados de seus produtos técnicos (ingredientes ativos) constituintes, optou-se por estimar a classe toxicológica de cada produto calculando a correspondente classe de periculosidade da OM S; para isso, utilizou-se a metodologia proposta pela própria $\mathrm{OM} \mathrm{S}^{2}$, que estabelece a classe de periculosidade com base na $\mathrm{DL}_{50}$ do(s) ingredientes ativos(s) e sua(s) concentração(ões) na formulação, estimando 0 valor da $\mathrm{DL}_{50}$ do produto comercial por meio da seguinte fórmula:

DL50 do ingrediente ativo $\times 100$ Porcentagem do ingrediente ativo na formulação

c) embora na legislação brasileira a classificação toxicológica possa ser definida por outros dados além da $\mathrm{DL}_{50}$, para fins deste estudo, no qual a idéia foi procurar mostrar o impacto potencial de uma mudança como a ocorrida nos critérios relacionados à $\mathrm{DL}_{50}$ para a classificação toxicológica, os dados de $\mathrm{DL}_{50}$ calculados pelo método da OMS foram utilizados para estimar as classes toxicológicas anteriores, definidas pela Portaria DISAD no $4 / 80^{7}$ e posteriores às mudanças de critérios, definidas pela Portaria SNVS no $3 / 92^{6}$;

d) para testar a concordância entre a classificação toxicológica anterior e a posterior à mudança de critérios, foi utilizado o programaSAS ${ }^{15}$ para o cálculo do coeficiente dekappa ${ }^{16}$, que pode variar de-1,00 a +1,00. Para interpretar a relação de concordância dos resultados foi empregada a escala proposta por Landis \& Koch ${ }^{17}$ :

$<0,00=$ ruim;

0,00 a $0,20=$ baixa;

0,21 a $0,40=$ regular;

0,41 a 0,60 = moderada;

0,61 a $0,80=$ substancial;

0,81 a $1,00=$ quase perfeita.

\section{Resultados}

AsFiguras 1 e2 comparam por meio de representação gráfica as mudanças provocadas pela regulamentação nos critérios que definem as faixas da classificação toxicológica dos agrotóxicos segundo a $\mathrm{DL}_{50}$ (toxicidade aguda), mostrando a sobreposição das faixas "antigas" com as "novas".

A Figura 1, referente às formulações líquidas, deixa claro a grande redução da amplitude da faixa que define a Classe I, de maior toxicidade, determinada pela modificação nos limites da faixa de classificação, redefinindo o restante dessa mesma faixa como Classe II. Os limites da antiga Classe II foram mantidos, porém reclassificando-a como ClassellI. Já a antiga Classe III foi incorporada pela Classe IV, ampliando, dessa forma, a classe de menor toxicidade.

A Figura 2 se refere às formulações sólidas. Neste caso, a antiga Classe I, de maior toxicidade, foi segmentada em três das classes atuais: Classes I, II ellI. As antigas Classes II ell I foram incorporadas pelas novas Classes III eIV, respectivamente, ampliando-as.

As Tabelas 1 a 3 apresentam os resultados obtidos na avaliação da estimativa do impacto da reclassificação toxicológica dos 495 produtos comerciais que estavam registrados em 1990.

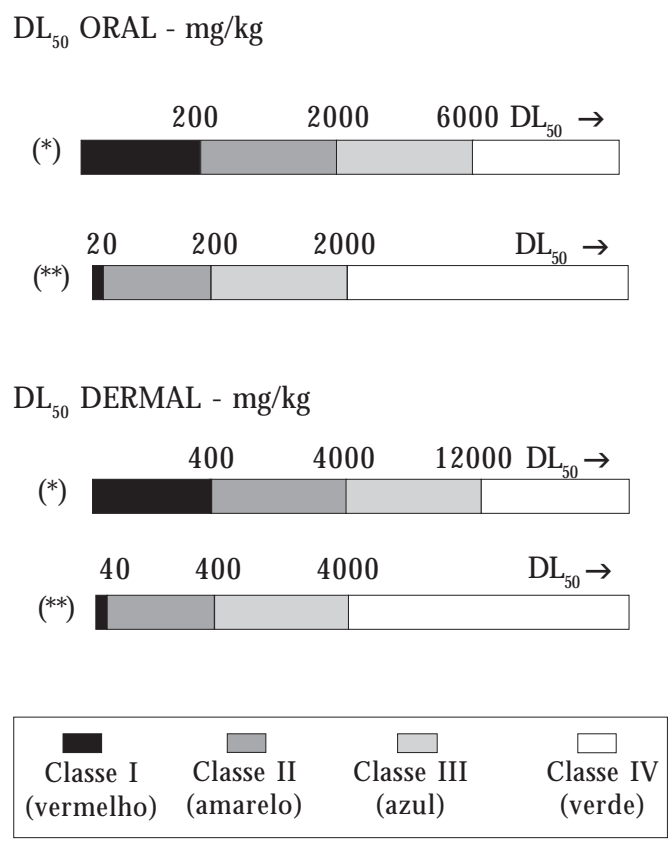

Figura 1. Comparação entre as faixas de classificação toxicológica das Portarias DISAD no 4/807 (*) e

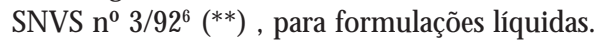




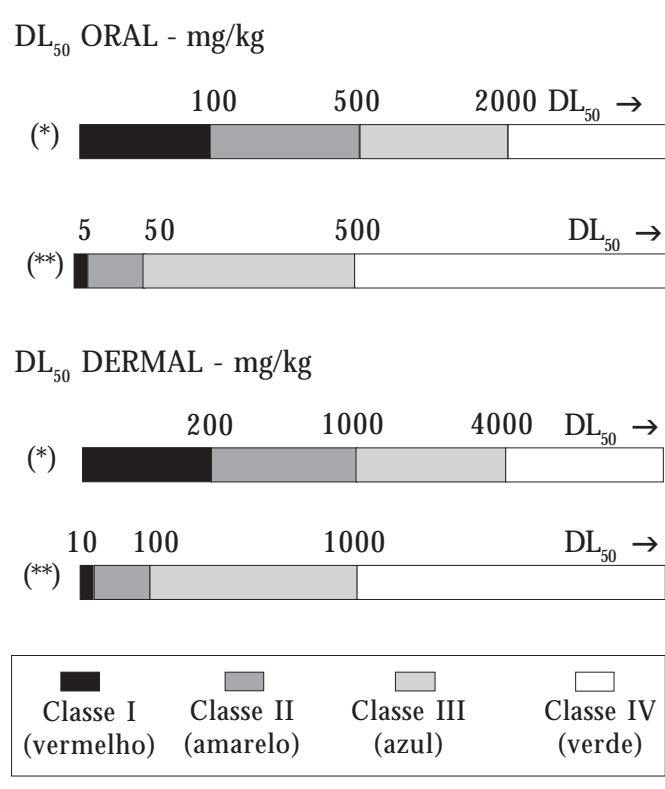

Figura 2. Comparação entre as faixas de classificação toxicológica das Portarias DISAD $n \cong 4 / 80^{7}(*)$ e SNVS nํ 3/92 $\left.{ }^{* *}\right)$, para formulações sólidas.
Tabela 2. Distribuição estimativa da classificação toxicológica* dos produtos comerciais com formulações líquidas que estavam registrados em 1990, segundo a concordância entre as classes toxicológicas definidas pela Portaria n 4/80 e pela Portaria n³/92.

\begin{tabular}{|c|c|c|c|c|c|}
\hline \multirow[b]{2}{*}{ Portaria n ${ }^{0} 3 / 92 * * *$} & \multicolumn{5}{|c|}{ Portaria n $4 / 80^{* *}$} \\
\hline & I & II & III & IV & Total \\
\hline I & 2 & 0 & 0 & 0 & 8 \\
\hline II & 31 & 0 & 0 & 0 & 31 \\
\hline III & 0 & 98 & 0 & 0 & 98 \\
\hline IV & 0 & 0 & 104 & 76 & 180 \\
\hline Total & 33 & 98 & 104 & 76 & 311 \\
\hline
\end{tabular}

* As classes toxicológicas dos produtos comerciais foram determinadas a partir da $\mathrm{DL}_{50}$ dos mesmos, calculada por metodologia da OMS?2.

** Classes toxicológicas, Portaria n 4/80: I - altamente tóxico; II - medianamentetóxico; III - pouco tóxico; IV - praticamente não tóxico.

*** Classes Toxicológicas, Portaria n 3/92: I - extremamente tóxico; II - altamentetóxico; III - medianamentetóxico; IV - pouco tóxico.

Fontes: Portaria DISAD n ${ }^{\circ} 4 / 80^{7}$ e Portaria SNVS $n^{\circ} 3 / 92^{6}$.
Tabela 1. Distribuição estimativa da classificação toxicológica* dos produtos comerciais que estavam registrados em 1990, segundo a concordância entre as classes toxicológicas definidas pela Portaria nº 4/80 e pela Portaria n 3/92.

\begin{tabular}{lccccc}
\hline & \multicolumn{5}{c}{ Portaria $\mathrm{n}^{\circ} 4 / 80^{* *}$} \\
\cline { 2 - 6 } Portaria n* 3/92*** & I & II & III & IV & Total \\
\hline I & 3 & 0 & 0 & 0 & 3 \\
II & 36 & 0 & 0 & 0 & 36 \\
III & 2 & 111 & 0 & 0 & 113 \\
IV & 0 & 0 & 141 & 202 & 343 \\
Total & 41 & 111 & 141 & 202 & 495
\end{tabular}

* As classes toxicológicas dos produtos comerciais foram determinadas a partir da $\mathrm{DL}_{50}$ dos mesmos, calculada por metodologiada OMS².

** Classes toxicológicas, Portaria n 4/80: I - altamente tóxico; II - medianamentetóxico; III - pouco tóxico; IV - praticamente não tóxico.

*** Classes toxicológicas, Portaria n 3/92: I - extremamente tóxico; II - altamentetóxico; III - medianamentetóxico;

IV - pouco tóxico .

Fontes: Portaria DISAD n ${ }^{\circ} 4 / 80^{7}$ e Portaria SNVS $n^{\circ} 3 / 92^{6}$.

Como pode ser observado na Tabela 1, estimou-se que, dos 41 produtos que pela $\mathrm{DL}_{50}$ poderiam estar classificados, antes das mudanças
Tabela 3. distribuição estimativa da classificação toxicológica* dos produtos comerciais com formulações sólidas que estavam registrados em 1990, segundo a concordância entre as classes toxicológicas definidas pela Portaria no $4 / 80$ e pela Portaria no 3/92.

\begin{tabular}{|c|c|c|c|c|c|}
\hline \multirow[b]{2}{*}{ Portaria n ${ }^{\circ} 3 / 92^{* * *}$} & \multicolumn{5}{|c|}{ Portaria n $4 / 80^{* *}$} \\
\hline & I & II & III & IV & Total \\
\hline I & 1 & 0 & 0 & 0 & 1 \\
\hline II & 5 & 0 & 0 & 0 & 5 \\
\hline III & 2 & 13 & 0 & 0 & 15 \\
\hline IV & 0 & 0 & 37 & 126 & 163 \\
\hline Total & 8 & 13 & 37 & 126 & 184 \\
\hline
\end{tabular}

* As classes toxicológicas dos produtos comerciais foram determinadas a partir da $\mathrm{DL}_{50}$ dos mesmos, calculada por metodologia da OM S2.

** Classes toxicológicas, Portaria $n^{\circ} 4 / 80$ : I - altamentetóxico; II - medianamentetóxico; III - pouco tóxico; IV - praticamente não tóxico .

*** Classes toxicológicas, Portaria no 3/92: I - extremamente tóxico; II - altamentetóxico; III - medianamentetóxico;

IV - pouco tóxico.

Fontes: Portaria DISAD n ${ }^{\circ} 4 / 80^{7}$ e Portaria SNVS $n^{\circ} 3 / 92^{6}$.

de 1992, na classe toxicológica I, apenas três se manteriam na mesma classe após a modificação da classificação, 36 passariam para a Classe II e2 
passariam para a Classe III. No caso dos produtos anteriormenteclassificadosna Classe II eClassellI, todos seriam reclassificados, passando respectivamente para a Classe III (111 produtos) e Classe IV (141 produtos). Ou seja, dos 495 produtos comerciais para os quais foi possível estimar a classe toxicológica anterior e posterior à alteração da classificação, 58,6\% podem ter sofrido reclassificação para classes toxicológicas consideradas de menor periculosidade. A plicando o coeficiente de kappa ${ }^{16,17}$, foi identificadauma bai xa concordância estatística entre a classificação anterior e a posterior à lei: $\mathrm{Kappa}=0,0780$; I.C. $95 \%[0,0427$ a 0,1132$] ; p=0,0042$.

D os 495 produtos comerciais analisados, 311 são formulações líquidas e 184, sólidas. Como os interval os que definem as faixas das classes toxicológicas são diferentes entre formulações líquidas e sólidas, o impacto da reclassificação de 1992 também foi estimado considerando separadamente os dados desses tipos de formulações.

A Tabela 2 apresenta os dados parciais referentes às formulações líquidas. Estimou-se que, dos 33 produtos que poderiam estar classificados, antes da mudança de 1992, na Classel, apenas dois teriam se mantido na mesma classe e 31 podem ter sido reclassificados para a Classe II. No caso dos produtos que anteriormente poderiam estar classificados na Classe II e Classe III, todos seriam reclassificados, passando respectivamente para a Classe III (98 produtos) e Classe IV (104 produtos). Ou seja, dos 311 produtos comerciais com formulações líquidas para os quais foi possível estimar a classe toxicológica anterior e posterior à mudança decritérios, 74,9\% podem ter sofrido reclassificação para classes toxicológicas consideradas de menor periculosidade. 0 coeficiente de kappa ${ }^{16,17}$ indicou que não há concordância estatística entre a classificação anterior ea posterior à lei: Kappa $=-0,0390 ;$ I.C. $95 \%$ [-0,0853 a 0,0073]; $p=0,2135$.

A Tabela 3 apresenta os dados parciais referentes às formulações sólidas. Dos oito produtos que poderiam estar classificados, antes da mudança de critérios, na Classe I, apenas um se manteria na mesma classe, cinco passariam para a Classe II e dois passariam para a Classe III. No caso dos produtos que anteriormente poderiam estar classificados nas Classe II eClassellI, todos seriam reclassificados, passando respectivamente para a Classe III (treze produtos) e Classe IV (37 produtos). Ou seja, dos 184 produtos comerciais com formulações sólidas para os quais foi possível estimar a classe toxicológica anterior e posterior à lei, $31,0 \%$ podem ter sofrido reclas- sificação para classes toxicológicas consideradas demenor periculosidade. 0 coeficientedekappa ${ }^{16}$, 17 indicou baixa concordância estatística entre a classificação anterior ea posterior à Lei: $K$ Kappa = 0,$1735 ;$ I.C. $95 \%$ [0,1049 a 0,2421]; $p=0,0003$.

\section{Discussão}

Inicialmente, faz-se necessário ressaltar que este trabalho sofreu limitações pela carência, desatualização, dispersão e discrepância dos dados necessários para o seu desenvolvimento. A falta de confiabilidade nesses dados para a finalidade pretendida levou à opção de se estimar, segundo a metodologia proposta pela $\mathrm{OM} \mathrm{S}^{2}$, a classificação toxicológica anterior e posterior às mudanças efetuadas pela regulamentação da "Lei dos Agrotóxicos" dos produtos que estariam registrados à época.

Antes da mudança promovida pela regulamentação da "Lei dos Agrotóxicos", os critérios de classificação toxicológica relacionados à toxicidade aguda empregados no Brasil estabeleciam faixas de maior amplitude que as sugeridas pela OM S para definir as classes de maior periculosidade à saúde. Por exemplo, no caso das formulações líquidas, ela praticamente ignorava a divisão da Classe I em Classe la e Ib proposta pela OMS, juntando-as em uma classe única, (Classe I - altamente tóxico). Mas, em 1992, a provável tentativa de harmonização com os critérios de classificação da OMS, estabelecida pela Portaria SN VS no $3^{6}$, do M inistério da Saúde, segundo as estimativas obtidas nestetrabal ho, pode ter provocado a reclassificação toxicológica, para classes de menor periculosidade, da maioria $(58,6 \%)$ dos produtos comerciais que se encontravam registrados naquele momento.

0 maior impacto deve ter ocorrido com as formulações líquidas, que, além de ser a forma de apresentação da maior parte dos produtos, de modo geral, por suas características de uso e capacidade de exposição, auferem maior risco ao trabalhador: apenas uma pequena parcela da antiga faixa da Classe I se manteve como tal, mudando a maior parte da faixa para a Classe II e as antigas faixas das Classes II e III mudaram, respectivamente, para III eIV. Pelos dados analisados, é possível estimar que cerca de $75 \%$ dos produtos registrados podem ter sofrido reclassificação com essas alterações.

No caso das formulações sólidas, as mudanças atingiram uma proporção menor de produtos, mas foram mais complexas. A antiga faixa 
da Classe I foi dividida em três classes, ficando parte na mesma Classe I, outra parte passou a ser Classe II e ainda uma terceira parte se transformou em ClassellI. Assim como ocorreu com as faixas das formulações líquidas, também nas sólidas as antigas faixas das Classes II e III se transformaram em III e IV, respectivamente. Com essas alterações, cerca de $31 \%$ dos produtos podem ter sido reclassificados.

Para agravar a situação, a Portaria no SNVS no $3 / 92^{6}$ manteve a relação entre o número da classe e a cor da faixa de identificação da classe toxicológica, mas modificou a relação entrea cor e a denominação da respectiva classe, implicando mais alterações na comunicação de riscos. As denominações empregadas anteriormente para designar as diferentes classes foram reaproveitadas em classes inferiores, acrescentando-se uma nova denominação para a Classe I, que embora tenha continuado associada com a faixa vermeIha do rótulo, passou a atribuir ao produto nela classificado a denominação de "extremamente tóxico". Jáa denominação "altamente tóxico", que antes dessa regulamentação denominava a Classe I, passou a ser empregada para a Classe II, na qual passou a ser associada com a faixa amarela. Assim, sucessivamente, o mesmo ocorreu com as denominações das classes subseqüentes, "me dianamente tóxico" e "pouco tóxico", que passaram a ser utilizadas nas classes de menor toxicidade, mudando do amarelo e azul, respectivamente, para azul everde.

Dessa forma, ao adotar as faixas da OMS, em 1992, a medida certamente criou confusão com a mudança das cores de sinal ização das faixas de advertência dos rótulos dos produtos que já estavam registrados, pois, embora o produto comercial fosse exatamente o mesmo, a mudança da cor eventualmente poderia ser interpretada pelo usuário como uma redução de toxicidade do produto. Essas modificações podem ter significado um agravamento nos impactos provocados pelo uso dos agrotóxicos, provocando, por exemplo, um aumento nas ocorrências de intoxicações, como sugerido por Poltroniéri ${ }^{18}$.

Isto mostra a importância de, previamente à adoção de mudanças, identificar eavaliar prováveis impactos provocados por alterações em critérios de classificação existentes.

Talvez um impacto imediato mais ameno pudesse ter ocorrido se o legislador não tivesse alterado as denominações e respectivas cores já consolidadas da classificação, adotando apenas uma faixa adicional com uma nova cor específica para a classe toxicológica intitulada "extrema- mente tóxico", estabelecendo assim uma classificação toxicológica com cinco faixas. Dessa forma, seguindo uma tendência contrária ao que ocorreu, haveria apenas o reenquadramento de alguns produtos da classe "altamente tóxico" na classeadicional sugerida, intitulada "extremamente tóxico", de maior periculosidade aguda à saúde. Com isso, provavelmente a maioria dos produtos poderia ter sido mantida nas classes anteriores, com as mesmas denominações e as mesmas cores nas faixas de advertência.

É preciso considerar também que classificar os produtos segundo a periculosidade dos mesmos, além de propiciar uma rotulagem adequada, com informações necessárias para alertar o usuário sobre os riscos na sua utilização, pode subsidiar a definição de medidas em diversos níveis, conforme preconizado por agências internacionais que têm interface com essa questão e discutido por Garcia ${ }^{19}$. Essas medidas podem incluir restrições de ordem técnica e administrativa para a indicação, a comercialização e o uso, auxiliando, por exemplo, na definição de taxações e controle de preços para os produtos mais tóxicos, ou influenciando na distribuição dos agrotóxicos, fazendo com que os produtos de maior periculosidade sofram restrições de uso e só possam ser utilizados sob determinadas circunstâncias ou por aplicadores especialmente capacitados, entre outras medidas que poderiam ser implantadas em âmbito federal, estadual e municipal. N esse sentido, o reenquadramento de um produto em classes de menor periculosidade pode restringir as possibilidades de aplicação demedidas degerenciamento de riscos como essas exemplificadas.

A implantação do GHS também poderá servir de subsídio para a proposição de medidas de gerenciamento de riscos. Levando em consideração a diversidade e quantidade de substâncias químicas produzidas e utilizadas (incluindo os agrotóxicos), os riscos à saúde e ao ambiente decorrentes dos processos de produção, transporte e uso de produtos químicos, a diversidade de sistemas de classificação e de comunicação de riscos existentes em vários países e a ausência de sistemas dessa natureza em algumas nações, 0 $\mathrm{GHS}^{8}$ propõe harmonizar a classificação de riscos visando à comunicação dos mesmos. Daí a relevância de sua adoção e implantação, no caso do Brasil, sobretudo pelo que a harmonização internacional pode ajudar na classificação de substâncias ainda não classi ficadas ena comunicação de riscos relacionados com o uso de produtos químicos em geral. 
Também no caso dos agrotóxicos, quejá contam com um sistema de classificação, mas que terá que ser reformulado para atender ao GHS, avanços deverão ser obtidos. Entretanto, se faz necessário considerar, de forma criteriosa e previamente à implantação, não apenas os aspectos positivos, mas também possíveis impactos negativos dessa readequação. Essa transição deve ser planejada e realizada de forma a evitar problemas na comunicação de riscos que possam confundir usuários e, conseqüentemente, implicar novos riscos.

\section{Colaboradores}

EG Garcia participou de todas as fases da pesquisa e de elaboração do texto. M A Bussacos trabalhou no delineamento, análise dos resultados, revisão e aprovação do texto. FM Fischer orientou o trabalho e participou da revisão e aprovação do texto. 


\section{Referências}

1. Copplestone JF. The development of the WHO Recommended Classification of Pesticides by $\mathrm{Ha}$ zard. Bulletin of the World Health Organization 1988; 66(5):545-551.

2. World Health Organization. The WHO recommended classification of pesticides by hazard and guidelines to classification 1990-1991. [documento na Internet]. [acessado 2000 mai 19]. International Programme on Chemical Safety - IPCS. Document WHO/PCS/90.1 Rev1. Disponível em: http:// whqlibdoc.who.int/hq/1990/WHO_PCS_90.1_ REV.1.pdf

3. Brasil. Ministério da Agricultura e Ministério da Saúde. Portaria no 220, de 14 de março de 1979. Disciplina rotulagem de defensivos agrícolas. In Gelmini GA, Novo JPS. Defensivos agrícolas: informações básicas e legislação. Campinas: Fundação Cargill; 1987. p. 436-445.

4. Brasil. Lei no 7.802, de 11 de julho de 1989. Dispõe sobre a pesquisa, a produção, a embalagem e rotulagem, o transporte, o armazenamento, a comercialização, a propaganda comercial, a utilização, a importação, a exportação, o destino final dos resíduos e embalagens, o registro, a classificação, o controle, a inspeção e a fiscalização de agrotóxicos, seus componentes e afins, e dá outras providências. In: M inistério da Agricultura e do Abastecimento. Legislação federal de agrotóxicos e afins. Brasília: M inistério da Agricultura e do Abastecimento; 1998. p. 7-13.

5. Garcia EG, Bussacos MA, Fischer FM. Impacto da legislação no registro de agrotóxicos de maior toxicidade no Brasil. Rev. Saúde Pública 2005; 39(5):832839.

6. Brasil. Portaria SNVS no 03, de 16 de janeiro de 1992. Ratifica os termos das "Diretrizes e orientações referentes à autorização de registros, renovação de registro e extensão de uso de produtos agrotóxicos e afins - no 1, de 09/12/91". In: Ministério da Agricultura e do Abastecimento. Legislação federal de agrotóxicos e afins. Brasília: M inistério da Agricultura e do Abastecimento; 1998. p. 153-177.

7. Brasil. Portaria DISAD № 4, de 30 de abril de 1980. Estabelece normas para a classificação toxicológica de defensivos agrícolas. In: Gelmini GA, Novo JPS. Defensivos agrícolas: informações básicas e legislação. Campinas: Fundação Cargill; 1987. p. 453-464.

8. United Nations Economic Commission for Europe. Globally Harmonized System of Classification and Labelling of Chemicals (GHS). [documento da Internet]. [acessado 2005 abr 12 ] [cerca de 16 p.]. Disponível em: http:// www.unece.org/trans/danger/ publi/ghs/ghs_welcome_e.html
9. World Health Organization. The WHO recommended classification of pesticides by hazard and guidelines to classification 2004. [documento da Internet]. International Programme on Chemical Safety - IPCS. [acessado 2006 Jun 27]. Disponível em: http:// www.who.int/ipcs/publications/pesticides_hazard_ rev_3.pdf.

10. Epi Info, version 6: a word-processing, database, and statistics program for public health on ibmcompatible microcomputers [programa de computador]. Versão 6.04b. Atlanta: Centers for Disease Control and Prevention; 1995.

11. Andrei E. Compêndio de defensivos agrícolas. $3 a$ ed. São Paulo: Andrei; 1990.

12. Agrofit 98. Informações de produtos fitossanitários registrados no MA [programa de computador]. Brasília (DF): Coordenação de Fiscalização de Agrotóxicos, M inistério da Agricultura e Abastecimento; 1998.

13. Agrotis. Sistema de Receituário Agronômico [programa de computador]. Versão 4.0. Curitiba (PR): Agrotis; 2000.

14. World Health Organization. The WHO recommended classification of pesticides by hazard and guidelines to classification 1998-1999. [documento da Internet]. International Programme on Chemical Safety - IPCS. Document WHO/PCS/90.1 Rev.1. [acessado 2000 mai 19]. Disponível em: http:// whqlibdoc.who.int/hq/1998//WHO_PCS_98.21.pdf

15. SAS Institute. SAS/STAT [programa de computador]. Release 8. N orth Caroline: SAS Institute; 1999.

16. Cohen J. A coefficient of agreement for nominal scales. Educ Psych M eas 1960; 20:37-46.

17. Landis JR, Koch GG. The measurement of observer agreement for categorical data. Biometrics 1977; 33:159-74.

18. Poltroniéri LC. O custo social do uso de praguicidas na agricultura por intoxicações: identificação de áreas de risco no Estado de São Paulo no período 1992/1994 [tese de livre-docência]. Rio Claro (SP): Departamento de Geografia, Instituto de Geociências e Ciências Exatas, UNESP; 1997.

19. Garcia EG. Segurança e saúde no trabalho rural: a questão dos agrotóxicos. São Paulo: Fundacentro; 2001.

Aprovado em 14/07/2007

Versão final em apresentada em 18/09/2007 